\title{
Telecom Network and Service Management: An Operator Survey
}

\author{
Stefan Wallin and Viktor Leijon \\ Luleå University of Technology LTU \\ SE-931 87 Skelleftea Sweden \\ stefan.wallin@ltu.se, leijon@csee.ltu.se
}

\begin{abstract}
It is hard to know which research problems in network management we should focus our attention on. To remedy this situation we have surveyed fifteen different telecom operators on four continents to gather some feedback on what they desire and expect from the network management research community. Their input forms a foundation for future directions in network management research, and provides us with valuable insight into what the most urgent problems are in industry.
\end{abstract}

\section{Introduction}

Network management research covers a wide range of different topics, and it is hard for the individual researchers to prioritize between them. One factor to take into account is the requirements emanating from the telecom industry.

In order to get an objective view of what the industry considers important we have surveyed fifteen different companies, to gather their opinions on the current state of network management systems, as well as their expectations on the future of Operational Support Systems (OSS).

As far as we can tell, there have been no previous surveys of this type for telecommunications network management. The process control and power industry areas seems to have a higher degree of industry feedback to the research 1223 , probably because of the human safety risks involved.

The results of this survey has strategic value both for researchers and solution vendors. It identifies areas where there is a strong need for further research and point to what changes are needed in order to stay competitive.

The contributions of this paper are:

- We present survey results from fifteen different companies, with a total of over 100 million customers, covering the current state (Section 3) and most important change drivers (Section 4).

- The respondents were then asked about their view on the future of OSS systems (Section 5) and what they expected from the OSS research community (Section 7.1).

- We conclude with a discussion of the focus areas identified in the survey: service topology and alarm quality in Section 7.2 


\section{Method}

We distributed the survey questions by e-mail to 20 operators of different sizes and on different continents. The individuals were selected based on their roles as network management architects or managers. 15 out of 20 operators answered. The respondents are a mix of fixed, broadband and mobile operators with a total customer base of over 100 million subscribers, see Table 1. The operators were classified by number subscribers into the categories $[<10 \mathrm{M},<20 \mathrm{M},<100 \mathrm{M}]$ to avoid giving out identifying information.

Some clarifying questions were sent over e-mail and a draft version of this paper was sent to all operators that provided answers. All questions except one were essay questions to avoid limiting the answers by our pre-conceived ideas. We have aggregated similar answers into groups, often using eTOM 4 processes as targets. The eTOM (enhanced Telecom Operations Map), published by the TM Forum, is the most widely used and accepted standard for business processes in the telecommunications industry. The operators were allowed to select several alternatives for every question.

Table 1. Summary of responding operators

\begin{tabular}{|l|l|l|}
\hline Services & Customers & Region \\
\hline \hline Mobile and Broadband & $<10$ million & North America \\
\hline Mobile & $<20$ million & Europe \\
\hline Mobile & $<10$ million & Europe \\
\hline Mobile & $<20$ million & South America \\
\hline Mobile & $<20$ million & North Africa \\
\hline Managed OSS, Mobile, transmission & $<20$ million & Europe \\
\hline Mobile, broadband, transmission & $<100$ million & Europe \\
\hline Mobile, broadband & $<10$ million & Europe \\
\hline Mobile, broadband, transmission & $<100$ million & Asia Pacific \\
\hline $\begin{array}{l}\text { Outsourced OSS, Mobile internet, broad- } \\
\text { band, 3G }\end{array}$ & $<10$ million & Europe \\
\hline Broadcast, virtual network, capacity & $<10$ million & Europe \\
\hline Mobile, broadband & $<10$ million & Europe \\
\hline Mobile, broadband, managed services & $<10$ million & Asia Pacific \\
\hline Full service carrier, wireline, wireless & $<10$ million & Asia Pacific \\
\hline Mobile and broadband & $<10$ million & Asia Pacific \\
\hline
\end{tabular}

\section{Current Status of Network Management}

The network and service management solution for a telecom operator is referred to as the OSS, the Operation Support System. We divide the OSS solutions into two types depending on maturity, either Classic or Advanced.

Classic OSS solutions covers service assurance and trouble ticketing. Element Managers are used to perform configuration management activities and performance management is only partially implemented. 
Advanced OSS solutions have expanded the solution to include service management tools such as active and passive probes; and service models. General configuration management tools are used to some degree, spanning several different vendors.

Common to both types is that security is not generally covered by the OSS solution. IT and Telecom is typically still split into different organizations and the IT department manages the customer care and billing processes.

All of the operators said that they focused on making the OSS a proactive solution, but admitted that in reality most of the OSS work is reactive, responding to alarms and problems reported to customer care. The proactive activities were typically based on probes and statistics, where the data was used to predict problems such as capacity limitations, but these were not really integrated into the overall OSS solution.

An interesting comment from one of the operators was about "OSS culture", they had problems transitioning their network administrators from using the element managers to using the full OSS solution. This resulted in underutilization of the OSS and decreased the motivation to develop it.

\section{OSS Motivation and Drivers}

We asked the operators to identify the most important external drivers that motivates changes in their OSS. The answers are shown in Figure 1 .

Increased focus on services and service quality was identified as the most important factor behind changes in the OSS. In order to understand this subject better we asked the operators to elaborate on how they viewed service management, one of the operators summarized it in the following way:

Services are not currently managed well in any suite of applications and requires a tremendous amount of work to maintain.

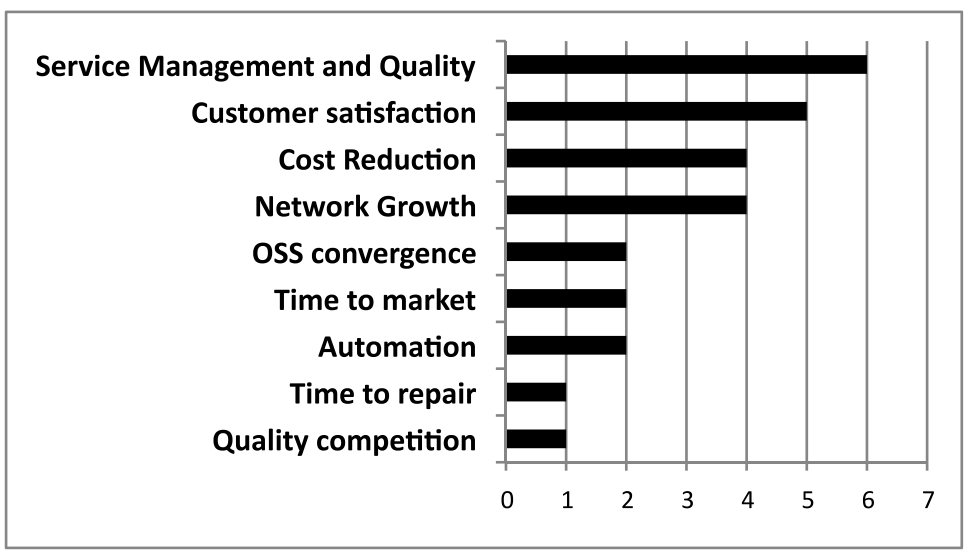

Fig. 1. Change Drivers for OSS 
The competitive market is pushing operators to offer more and more innovative services, including SLAs, which require the OSS solution to measure the service quality.

One operator described the experience with the two major alternatives to service monitoring, either using probes or mapping existing events and alarms to a service model. The latter approach failed since there was no good way to describe which alarms were really critical. They made the decision to use only probing for future services, stressing that future services will have service probing from the service deployment phase.

Some of the operators stressed the importance of standards for service models. The problem with models is that services are rapidly changing, therefore requiring a large amount of customization work. One operator expressed reservations about how detailed services can be:

Time and money will not be available to [develop] sophisticated approaches over a long period. Customers will have to accept limited quality assurance and quality documentation. Service levels will always be high level, if [they exist] at all.

Another operator commented on how the use of service models is evolving:

Service models are becoming more and more important: currently [they are] not implemented in core processes but used as means to semi-document and analyze when evaluating impact of faults, new services, etc.

As indicated by Figure 1 cost reduction is clearly another key factor. We asked the operators to further break down the cost drivers and the results are shown in Figure 2 .

The first two items can be considered two sides of the same coin: Integration costs are high in OSS due to the introduction of new technologies and services. When a new type of network element is deployed it needs to be integrated into the OSS solution and while most solutions are based on well established products,

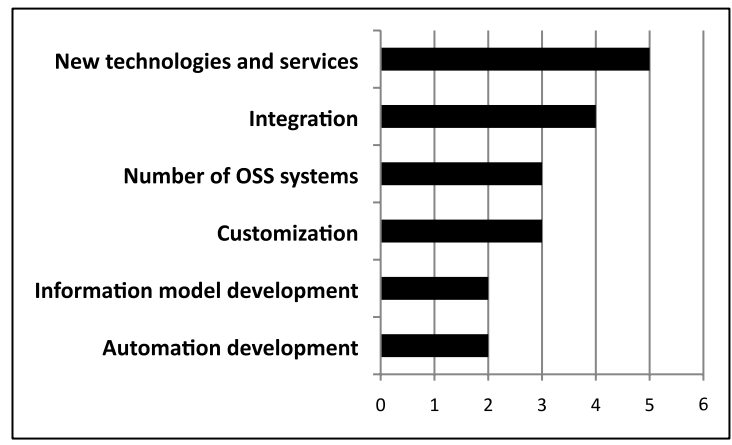

Fig. 2. Cost drivers for OSS 
there is still a high degree of customization needed to adopt the tools to user needs and processes.

In order to get a unified view of the solution, the resource oriented interfaces needs to be mapped into an overall service model. Operators are struggling with this challenge, the "information model development" in Figure 2, Finally the OSS itself is expensive due to the number of components that are needed. Even in the case where an OSS is built using only one vendor, it is still made up of a portfolio of modules which add up to a relatively costly software solution.

Returning to the change drivers (Figure10 the next items are network growth and increased focus on customer satisfaction. While network growth is inherent in network management, customer satisfaction has not historically been a primary goal for OSS solutions.

To put the true business role of the OSS solution in focus, we asked if it was seen as a competitive tool or not. The answers were divided into two general streams:

- Yes. Motivations are the desire to decrease time-to-repair, decrease OPEX, and improve customer satisfaction by quicker response to new requirements and customer complaints. Two thirds of the responses fall into this category.

- No. These operators felt that it will be outsourced. The out-sourcing scenario was partly motivated by internal failures and a desire to give the problem to someone else.

\section{The Future of OSS}

\subsection{Organizational Changes}

The answers regarding future OSS changes are summarized in Figure 3 , it is no surprise that Service management is seen as one of the most important upcoming changes. One of the larger operators predicted a "focus on service management - bringing this up to $40 \%$ from [the] current level of $5-10 \%$ ".

Further, we see the strategic need for a service inventory to enable service quality, service provisioning and a service life-cycle view. Most of the operators are providing broadband services and this makes automatic service provisioning a must.

Service management was summarized in the following way by one of the operators:

Managing services must be the focus of the future development, while pushing network management into a supporting role, [...] service models [should be] constructed from auto-discovered infrastructure components that have auto-discovery deeply integrated. [...] [The] service models should be abstracted from physical and logical inventory to ensure there is a life cycle. [...] If the service models were to be constructed from scratch, with no base layer that is managed separately (auto-discovered or abstraction over inventory), that will most probably undermine the layered service level management solution. 


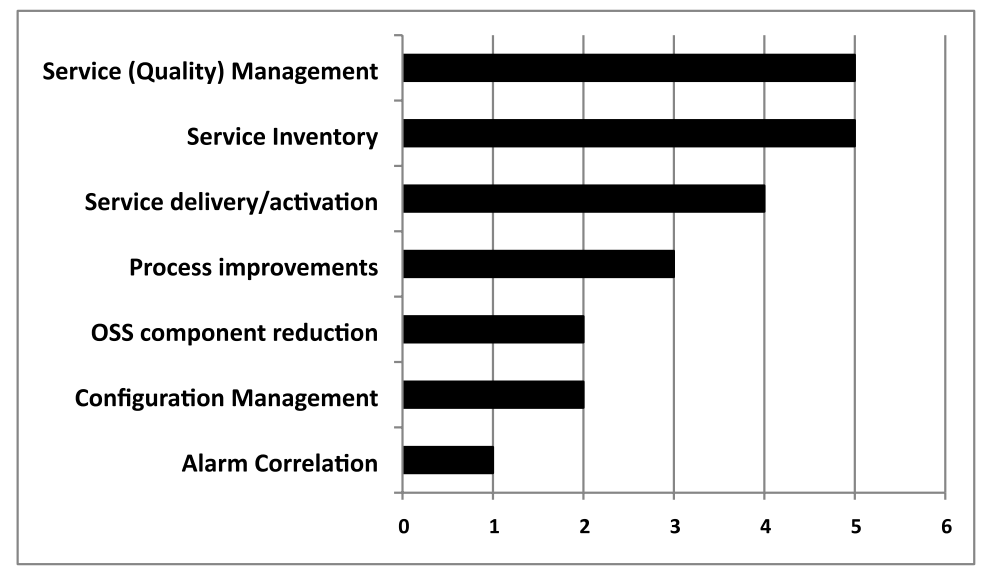

Fig. 3. OSS organizational changes

Common to all respondents is an increased focus on customer care, customer service, self-management, and self-provisioning. An interesting point made by one of the operators is the shift from eTOM verticals to a more supply chain based production model. Classical OSS systems have had separate solutions for fulfillment, assurance, and billing. The supply chain model will focus on the whole life-cycle from service definition to billing and retirement. This will remove the obstacles between eTOM verticals and help relieve the hand-over problem from "eTOM Strategy" to "eTOM Operations" areas.

Another trend is a radical transformation of the OSS from being a networkoriented function to becoming a customer-oriented organization. The surveyed operators mentioned features such as "more focus on customer service, customer experience, and self-management".

\subsection{Focus Processes}

The eTOM process framework defines the processes in several abstraction layers. We asked the operators to identify the three most important processes (Figure 4).

The prefixes of the legend in Figure 4 refers to the eTOM vertical (Fulfillment, Assurance, Billing) and horizontal (Customer, Service, Resource, Supplier) endto-end processes. For instance F-S refers to Fulfillment-Service. Again, we see the focus on the service life-cycle through order handling, provisioning, service quality, problem management and billing.

\section{Standards and Research Efforts}

\subsection{Standards}

The attitude towards standards was not very enthusiastic: "They are too complicated and are actually adding to the cost of ownership", in this case the 3GPP 


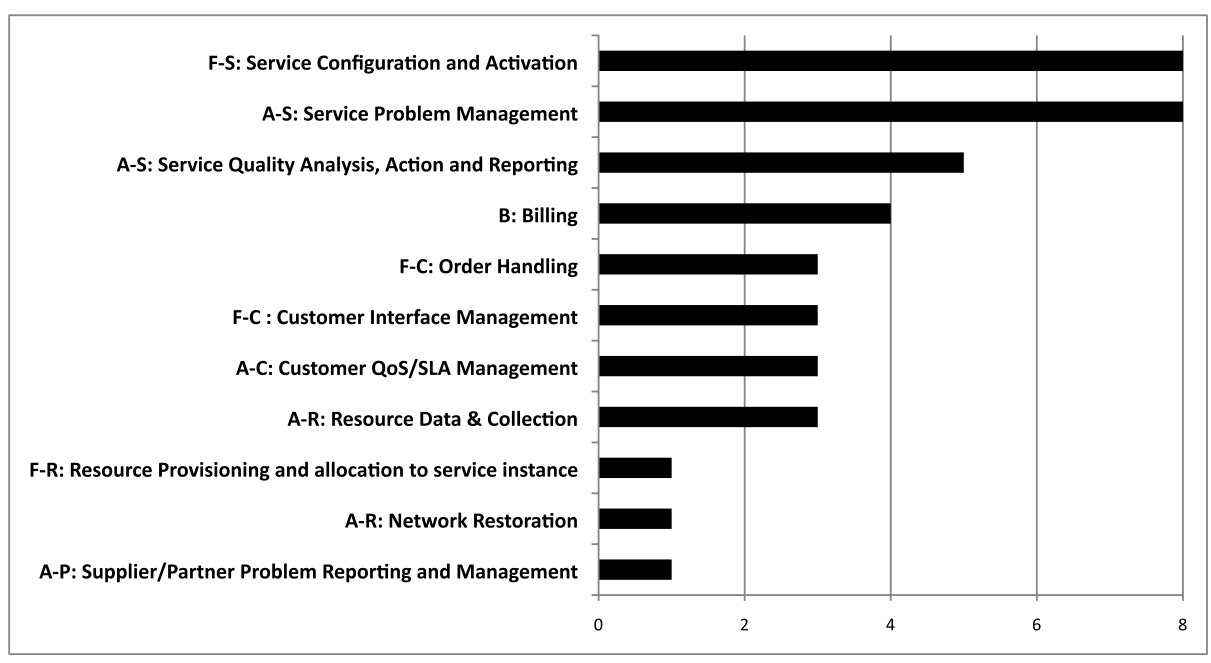

Fig. 4. The most important eTOM processes

Alarm Interface was the main source for concern. Another operator had similar distrust for standards: "[In] alarm integration to the OSS, most of the vendors do not follow any one. We are pushing [our] internal standard to have useful alarm information for the end users". Some operators mentioned SNMP as a working protocol that is easy to integrate, however the lack of standard OSS interface MIBs is a problem, and the vendor MIBs vary in quality.

As important areas for future standardization efforts they mentioned "interfaces, data and semantic models, standardization of procedures" and "well defined top level common framework and common languages". We see from these comments that the current practice of using different protocols for different interfaces and having weak formal data models is a problem for OSS integrations. There is no accepted overall common framework which would enable unified naming of resources.

Surprisingly, none of the operators mentioned OSS/J [5]. On the other hand several operators considered the eTOM [4] and ITIL [6] process standards to have real practical value. They used these process frameworks to structure the work and make it more efficient.

\subsection{Research Efforts}

In Figure 5 we can see the areas that the operators in this survey identified as the most important ones for future research.

It is well worth noting that for research alarm correlation is a key item, while it was not as prominent when the operators were asked to identify the key efforts and changes in the coming years. The opposite is true for service management and modeling, which were key items for more immediate change. One interpretation is 


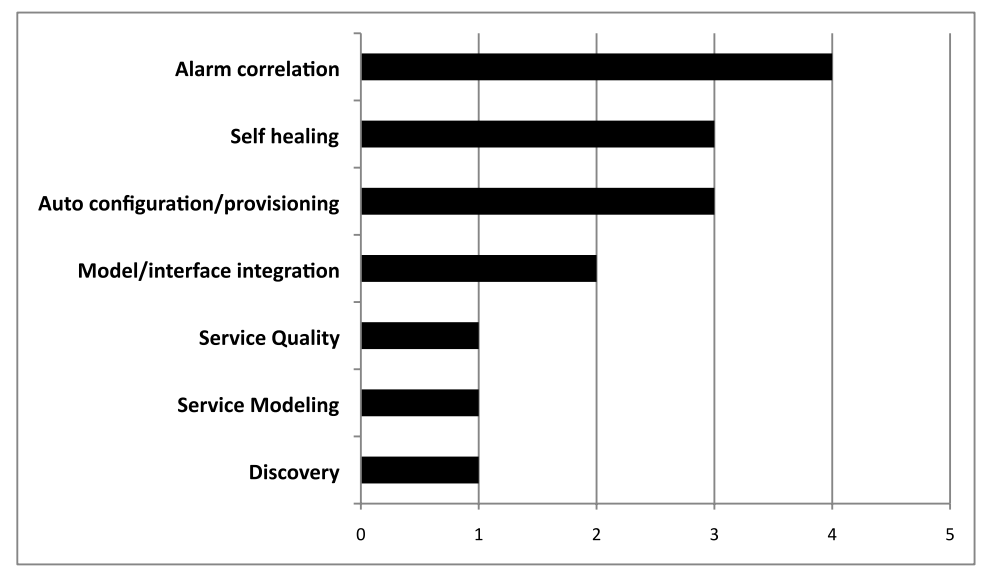

Fig. 5. Research efforts

that it is hard to foresee the challenges with the planned changes before we have attempted them. Many operators have struggled with alarm correlation for years without seeing any clear successes and are hoping for more research to help them. We might see the same thing happening with service management and modeling after a first wave of implementation efforts.

\section{Discussion}

\section{$7.1 \quad$ OSS Research}

Historically a lot of research has gone into alarm correlation. Looking at the current correlation rates it is not clear how successful these projects have been, many correlation projects are facing challenges trying to capture operator knowledge and transforming it into rules. Other methods for finding rules based on rule discovery, knowledge management, data-mining and self-learning networks are interesting, but seem to require further investigation to be of practical use.

Having service models in place would be a key to unlocking many other functions. The current industry practice of large UML models with loose structure does not seem able to cope with the requirements, more modular and semantically rich ways of doing service modeling are required.

Another basic area is that of formal interface definitions. The current integration costs can not be justified when we consider the fundamentally simple nature of the information that flows over the interfaces, integrating alarms should not be a complex and costly task. Semantically richer interface definitions would probably improve the situation, but this requires a focus on the semantics of the model and not only the syntax, protocol and software architecture.

Boutaba and Xiao [7] point to the following major enablers for future OSS systems: 
- Policy-based network management, 8], 9]

- Distributed computing, [10], [11]

- Mobile agents, 12, 13

- Web techniques, 14

- Java, 15

These are topics that we see in many network management efforts. However we see little correlation between these and those identified in this survey. Furthermore it partly illustrates the research's focus on software architectures rather then management.

\subsection{Fulfilling the Future Needs of Operators with OSS}

It is easy to talk about paradigm shifts but things change more slowly than we expect, nonetheless some major changes in OSS solutions are needed to help service providers to cope with the changing environment. Operators want to manage services rather than the network resources that are used to deliver services. This change in focus is driven by several factors; increased competition, more complex service offerings, distribution of services, and a market for Service Level Agreements.

One operator had this to say about the market for services:

The competition between companies pushes them to offer more and innovative services, they need to sell more services first, the want to go out before the others.

Current network management solutions control and administer resources; physical resources are found, configured and monitored. However, from a customer point of view, services are bought, provisioned and billed.

One of the underlying challenges in a service centric solution is to maintain a model of the service topology. In contrast to the network resources services are abstract by nature, while it is often possible to automatically discover the network topology this is not necessarily true for the service topology.

Future solutions must rely on service repositories where different and systems publish their respective topology information with lightweight technologies, this will enable operators to maintain a centralized view of the topology which will be the future OSS foundation. Note that this improvement has to come without significant additional integration costs or complexity.

Moving forward in the direction identified in this survey will fail if there is no way to share the information model between OSS systems. The feedback we get from operators who apply SID [16] is that it works well as a design base for the OSS system but not as a service model to maintain the dynamic business services. Service modeling must be dynamic and have well-defined semantics, the current practice of static models and informal documents does not cope with a changing environment.

While OSS solutions have primarily been network oriented it now needs to change focus to customer care, since operators see a huge possibility to reduce 
costs in an automated customer care. The number of employees in mobile network customer care greatly outnumbers the OSS staff, therefore, solutions that help automate customer care activities will be a priority in the coming years.

Operators will also look for automated provisioning solutions including selfconfiguration of the network elements which helps avoid tedious parameter setting. The move from fixed-line services to broadband consumer services stresses this further since customers need to be able to buy, configure and troubleshoot their services with minimal support.

While we see these changes coming we need to realize that the integration of Telecom, IT and IP has not yet happened. Some of the operators have a somewhat naïve vision of a future when network administrators will only look at service views and SLA status.

None of our respondents reported positive results regarding the deployment of standards. Over the years we have seen great efforts to move from one protocol to another, from OSI-based solutions to CORBA and now Web Services. This journey is based on a desire to find a technology solution to an information problem, unfortunately OSS integration standards like OSS/J has not yet proven its cost effectiveness.

Alarm quality and alarm correlation is still an underdeveloped area, although research and industry initiatives go back decades [17] the current alarm flow at a standard network operations centre is fairly basic and often of low quality.

We did not get any real numbers on filtering and correlation rates, but the informal indications pointed to very low success rate which is consistent with what is reported by Stanton [18. In many cases alarm messages go untransformed, unfiltered, and uncorrelated from the network elements to the network management system which leads to a chaotic situation that needs to be cleaned up before we can move into service and customer management. A representative answer was:

[around] $40 \%$ percent of the alarms are considered to be redundant as many alarms appears at the same time for one 'fault'. Many alarms are also repeated [...]. One alarm had for example appeared 65000 times in todays browser. Correlation is hardly used even if it supported by the systems, [current correlation level is] 1-2\% maybe.

Some operators chose to completely ignore alarm correlation, they considered it too expensive and complex to get good results. These respondents instead pointed to probing, statistics, and performance based solutions to get an overall picture rather then trying to automate root-cause analysis. It was also stressed that advanced alarm correlation projects are in many cases signs of bad alarm quality from the low-level systems.

Finally, we let an operator conclude this survey by pointing to ongoing challenging OSS improvements:

Significant work underway to move to a self-service enabled environment for the customer. Whilst this is to improve the customer experience, this is also expected to dramatically increase operational efficiencies. Other 
key improvements are, (1) [...] capability to enable growth and the rapid on-boarding of customers, (2) Improve flow-through provisioning and activations [...] to reduce manual intervention, improve service delivery timeframes and room for human error.

\section{Conclusion}

We hope that this survey can form the basis for prioritizing among research topics. The most important conclusion is probably that there is a great potential to further network management research by working closer with service providers. There is a gap between the current research efforts which typically focus on new software architectures and protocols and the telecom companies that has other priorities.

It is worth noting that after decades of research, alarm correlation is still the most prioritized research area. This can partially be interpreted as a failure, since no solution seems to be ready. Instead we see a new set of research challenges emerging, connected to self-healing, service activation and provisioning.

If research is to support the future focus areas for service providers we need to find solutions for service and quality management. Another observation is the failure of alarms as an indicator of service status, where we see a trend towards probe based solutions.

The operators gave a clear message on their desire to move from network and resource management towards customer and service management solutions. This comes as no surprise, as the trend has been clear for some time, but the path there needs attention. A new brand of OSS Solutions that are based on the service life-cycle rather then separate OSS components for different processes is needed.

\section{References}

1. Bransby, M., Jenkinson, J.: The Management of Alarm Systems. HSE Contract Research Report 166, 1998 (1998)

2. Stanton, N.: Human Factors in Alarm Design. Taylor \& Francis, Abington (1994)

3. Hollifield, B., Habibi, E.: Alarm Management Handbook. PAS (2006)

4. TMForum: eTOM - Telecom Operators Map (2006)

5. TM Forum: OSS/J, http://www.tmforum.org/ossj/ (accessed August 14, 2008)

6. Office of Government Commerce: ITIL, http://www.itil-officialsite.com (accessed August 14, 2008)

7. Boutaba, R., Xiao, J.: Network Management: State of the Art. In: Communication Systems: The State of the Art: IFIP 17th World Computer Congress, TC6 Stream on Communication Systems, Montréal, Québec, Canada, August 25-30 (2002)

8. Strassner, J.: Policy-Based Network Management: Solutions for the Next Generation. Morgan Kaufmann, San Francisco (2004)

9. Lymberopoulos, L., Lupu, E., Sloman, M.: An Adaptive Policy-Based Framework for Network Services Management. Journal of Network and Systems Management 11(3), 277-303 (2003) 
10. Tennenhouse, D., Wetherall, D.: Towards an active network architecture. In: DARPA Active Networks Conference and Exposition, Proceedings, pp. 2-15 (2002)

11. Chen, T., Liu, S.: A model and evaluation of distributed network management approaches. IEEE Journal on Selected Areas in Communications 20(4), 850-857 (2002)

12. Papavassiliou, S., Puliafito, A., Tomarchio, O., Ye, J.: Mobile agent-based approach for efficient network management andresource allocation: framework and applications. IEEE Journal on Selected Areas in Communications 20(4), 858-872 (2002)

13. Stephan, R., Ray, P.: Network Management Platform Based on Mobile Agents. Int. J. Network Mgmt. 14, 59-73 (2004)

14. Pavlou, G., Flegkas, P., Gouveris, S., Liotta, A.: On management technologies and the potential of Web services. IEEE Communications Magazine 42(7), 58-66 (2004)

15. Lee, J.: Enabling network management using Java technologies. IEEE Communications Magazine 38(1), 116-123 (2000)

16. TM Forum: Shared information data model (2005)

17. Steinder, M., Sethi, A.S.: A survey of fault localization techniques in computer networks. Science of Computer Programming 53(2), 165-194 (2004)

18. Stanton, N., Harrison, D., Taylor-Burge, K., Porter, L.: Sorting the Wheat from the Chaff: A Study of the Detection of Alarms. Cognition, Technology \& Work 2(3), 134-141 (2000) 